\title{
ARITHMETIC SUBGROUPS OF ALGEBRAIC GROUPS
}

\author{
BY ARMAND BOREL AND HARISH-CHANDRA
}

Communicated by Deane Montgomery, July 22, 1961.

A complex algebraic group $G$ is in this note a subgroup of $G L(n, C)$, the elements of which are all invertible matrices whose coefficients annihilate some set of polynomials $\left\{P_{\mu}\left[X_{11}, \cdots, X_{n n}\right]\right\}$ in $n^{2}$ indeterminates. It is said to be defined over a field $K \subset C$ if the polynomials can be chosen so as to have coefficients in $K$. Given a subring $B$ of $C$, we denote by $G_{B}$ the subgroup of elements of $G$ which have coefficients in $B$, and whose determinant is a unit of $B$. Assume in particular $G$ to be defined over $\boldsymbol{Q}$. Then $G_{\boldsymbol{Z}}$ is an "arithmetically defined discrete subgroup" of $G_{R}$, or, more briefly, an arithmetic subgroup of $G_{R}$. A typical example is the group of units of a nondegenerate integral quadratic form, and as a matter of fact, the main results stated below generalize facts known in this case from reduction theory. The proofs will be published elsewhere.

1. Reductive groups. A complex algebraic group $G$ is an algebraic torus (a torus in the terminology of [1]) if it is connected and can be diagonalized or, equivalently, if it is birationally isomorphic to a product of groups $C^{*}$ [1, Chapter II]. The group $G$ is reductive if its identity component $G^{0}$ may be written as $G^{0}=T \cdot G^{\prime}$, where $T$ is a central algebraic torus, and $G^{\prime}$ is an invariant connected semi-simple group, or, equivalently, if all rational representations of $G$ are fully reducible.

LEMma 1. Let $G_{1} \supset \ldots \supset G_{m}$ be reductive algebraic subgroups of $G L(n, C)$, defined over $R$. Then there exists $a \in S L(n, R)$ such that the groups $a \cdot G_{i R} \cdot a^{-1}$ are stable under $x \rightarrow^{t} x(i=1, \cdots, m)$.

This lemma, formulated in a somewhat different terminology, is due to G. D. Mostow [4]. Lemma 1, for $m=1$, implies easily that the usual properties of maximal compact subgroups and of the Iwasawa decompositions (see [7] for instance) are valid for real algebraic reductive groups.

LEMMA 2. Let $G$ be a connected reductive complex algebraic group, $H$ an algebraic subgroup. Then $G / H$ is an affine variety if and only if $H$ is reductive. If $G$ and $H$ are defined over $Q$, and $H$ is reductive, there exists a rational representation $\pi: G \rightarrow G L(m, C)$, defined over $\boldsymbol{Q}$, such 
that there is a point $v \in Z^{m}$ whose orbit under $G$ is closed and whose isotropy group is $H$.

The fact that if $G / H$ is an affine variety (or more generally a Stein manifold) then $H$ is reductive, is due to Matsushima [3] (whose proof can be simplified using ordinary cohomology with complex coeffcients). The converse is stated in [3] (and attributed to Iwahori and Sigiura); it can be proved by realizing $G / H$ as a closed orbit in a suitable linear representation.

2. Siegel domains. Let $G \subset G L\left(n, C_{i}\right)$ be an algebraic reductive group defined over $R, G_{R}=K \cdot A \cdot N$ an Iwasawa decomposition of $G_{R}$, and $\mathfrak{g}, \mathfrak{f}, \mathfrak{a}, \mathfrak{n}$ the Lie algebras of $G_{R}, K, A, N$ respectively. $K$ is a maximal compact subgroup, $A$ is real diagonalizable, connected, $N$ is unipotent, $\mathfrak{a}$ is orthogonal to $\mathfrak{l}$ with respect to the Killing form. Let further $\psi \subset \mathfrak{a}^{*}$ be the set of roots of $\mathfrak{g}$ with respect to $\mathfrak{a}$ (the restricted roots) and, for $\alpha \in \psi$, let $\mathfrak{g}_{\alpha}=\{x \in \mathfrak{g},[h, x]=\alpha(h) \cdot x, h \in \mathfrak{a}\}$. Then, for a suitable ordering on $\mathfrak{a}^{*}$, we have $\mathfrak{n}=\sum_{\alpha>0} \mathfrak{g}_{\alpha}$. Let $A_{t}$ $=\{a \in A, \alpha(\log a) \leqq t, \alpha \in \psi, \alpha>0\},(t>0)$. A Siegel domain of $G_{R}$, with respect the given Iwasawa decomposition, is a subset $\mathfrak{S}_{t, \infty}$ $=K \cdot A_{t} \cdot \omega$, where $\omega$ is a compact set in $N$. The $\Im_{t, \omega}$ 's, ordered by inclusion, form a filtered set, and their union is $G_{R}$. One would obtain equivalent families by letting $\alpha$ run only through the simple restricted roots in the definition of $A_{t}$, or by replacing $A_{t}$ by $a_{0} \cdot A^{-}\left(a_{0} \in A\right)$, $A^{-}$being the exponential of the negative Weyl chamber. It is easily seen that if $G$ is semi-simple, a Siegel domain has finite Haar measure.

A standard Siegel domain $\mathfrak{S}$ in $G L(n, R)$ is a Siegel domain with respect to the usual Iwasawa decomposition (where $K=O(n), A$ is the group of diagonal matrices with strictly positive entries, $N$ the group of upper triangular unipotent matrices) such that $G L(n, R)$ $=\subseteq \cdot S L(n, Z)$. The existence of such domains is classical. By a well known theorem of Siegel [8], given $x \in G L(n, Q)$, the set of $y \in S L(n, Z)$ such that $\subseteq \cap \Im \cdot y \cdot x \neq \varnothing(\operatorname{resp}$. $\subseteq \cap \Im \cdot x \cdot y \neq \varnothing)$ is finite. $\subseteq \cap S L(n, R)$ will be called a standard Siegel domain of $S L(n, R)$.

LEMMA 3. Let $\pi: S L(n, C) \rightarrow G L(m, C)$ be a right rational representation, defined over $\boldsymbol{Q}, v \in \boldsymbol{R}^{m}$ be a point whose orbit under $S L(n, R)$ is closed and whose isotropy group in $S L(n, R)$ is stable under $x \rightarrow t x$, and let $\mathfrak{S}$ be a standard Siegel domain of $S L(n, R)$. Then $v \cdot \pi(\Im) \cap Z^{m}$ is finite.

This lemma can be proved more generally for a rational representation of a reductive group, a suitable Iwasawa decomposition and a Cartan involution compatible with it, but the above special case 
suffices for the applications. Applied to the natural representation of $S L(n, C)$ in the space of quadratic forms, it yields the finiteness of the number of reduced integral forms with a given nonzero determinant, stated first by Hermite [2].

3. Fundamental sets for arithmetic subgroups. As is well known, Hermite has used the result just mentioned to construct a fundamental set for the group of units of a nondegenerate quadratic form $F$ in the space of majorizing forms of $F[2]$. The construction of the set $U$ below is in a sense a generalization of his procedure.

TheOREM 1. Let $G$ be a connected complex algebraic group defined over 8 . Then there exists an open set $U$ in $G_{R}$ with the following properties: (i) $G_{R}=U \cdot G_{Z}$; (ii) $K \cdot U=U$ for a suitable maximal compact subgroup of $G_{?}$; (iii) For any $x \in G_{Q}, U^{-1} \cdot U \cap\left(x \cdot G_{Z} \cup G_{Z} \cdot x\right)$ is finite; (iv) if $G$ has no nontrivial rational character defined over $Q, U$ has finite Haar measure.

The group $G$ is the semi-direct product of a reductive group and an invariant unipotent group $N$, both defined over $\boldsymbol{Q}$. Since $N_{R} / N_{Z}$ is compact, the proof of Theorem 1 is easily reduced to the case where $G$ is reductive. Assuming moreover, as we may, $G \subset S L(n, C)$, we take a right rational representation $\pi: S L(n, C) \rightarrow G L(m, C)$ such that $\pi(S L(n, Z)) \subset S L(m, Z)$, and that there exists $v \in Z^{m}$ whose orbit is closed and whose isotropy group is $G$. The existence of $\pi$ follows mainly from Lemma 2. Let $a \in S L(n, R)$ be such that $a \cdot G_{R} \cdot a^{-1}$ is stable under $x \rightarrow^{t} x$ (Lemma 1) and $\subseteq$ be an open neighborhood of a standard Siegel domain of $S L(n, R)$ contained in a standard Siegel domain. By Lemma 3, there exists a finite number of elements $b_{1}, \cdots, b_{m} \in S L(n, Z)$ such that

$$
v \cdot \pi(S L(n, Z)) \cap v \cdot \pi\left(a^{-1} \subseteq\right)=\left\{v \cdot \pi\left(b_{1}^{-1}\right), \cdots, v \cdot \pi\left(b_{m}^{-1}\right)\right\} .
$$

We have $G_{R}=a^{-1} \cdot H$, where $H$ is the set of elements in $S L(n, R)$ which map $v \cdot \pi\left(a^{-1}\right)$ onto $v$. From this, (1) and the equality $S L(n, R)$ $=\mathfrak{S} \cdot S L(n, Z)$ it follows easily that $U=U_{i}\left(G_{R} \cap a^{-1} \cdot \mathfrak{S} \cdot b_{i}\right)$ satisfies (i), (ii). Property (iii) is then a consequence of the theorem of Siegel recalled in $\$ 2$. When $G$ is semi-simple, property (iv) follows from the following lemma:

Lemma 4. Let $G_{1} \subset G$ be algebraic semi-simple groups. Assume that there are Irwasawa decompositions $G_{1 R}=K_{1} \cdot A_{1} \cdot N_{1}, G_{R}=K \cdot A \cdot N$ of $G_{1 R}$ and $G_{R}$ such that $K_{1} \subset K, A_{1} \subset A, N_{1} \subset N$, and that a positive root of the Lie algebra of $G_{R}$ with respect to the Lie algebra of $A$, for the ordering defined by $N$, restricts to a positive root of the Lie algebra of $G_{1 R}$, for 
the ordering defined by $N_{1}$, Let $\subseteq$ be a Siegel domain of $G_{R}$ with respect to the Irvasawa decomposition $K \cdot A \cdot N$, and $x \in G_{R}$. Then $\subseteq \cdot x \cap G_{1_{R}}$ is contained in a finite number of translates of a Siegel domain of $G_{1 R}$.

By an elementary argument, property (iii) implies the

CoRollaRy. Let $G$ be a complex algebraic group defined over $\mathbf{Q}$. Then $G_{Z}$ is finitely generated.

The result on reduced forms stated at the end of $\$ 2$ implies the finiteness of the number of classes of integral forms with a given nonzero determinant. The latter has the following generalization.

Theorem 2. Let $G$ be a connected reductive algebraic group defined over $\boldsymbol{Q}$, and $\pi: G \rightarrow G L(m, C)$ a rational representation defined over $\boldsymbol{Q}$, and $H=G_{\boldsymbol{Z}} \cap \pi^{-1}(\boldsymbol{G L}(m, \boldsymbol{Z}))$. Then for any closed orbit $X$ of $G$ in $\boldsymbol{C}^{m}$, the integral points of $X$ form a finite number of orbits of $H$.

Corollary. Let $G, G^{\prime}$ be connected algebraic groups, defined over $\mathbf{Q}$, and $\mu: G \rightarrow G^{\prime}$ a rational surjective homomorphism with finite kernel, defined over $\boldsymbol{Q}$ (an isogeny). Then $\mu\left(G_{z}\right)$ and $G_{z}^{\prime}$ are commensurable.

4. Arithmetic subgroups with compact fundamental sets. The quotient $G_{R} / G_{Z}$ is compact for instance when $G$ is the orthogonal group of a form which does not represent zero. This fact has the following generalization, which had been conjectured by R. Godement:

TheOREM 3. Let $G$ be a complex algebraic group defined over $Q$. Then the following conditions are equivalent: (i) $G_{R} / G_{Z}$ is compact: (ii) the identity component of $G$ has no nontrivial rational character defined over $\boldsymbol{Q}$, and every unipotent element of $G_{\mathcal{Q}}$ (or, equivalently, of $G_{Z}$ ) belongs to the radical of $G_{Q}{ }^{1}$

Remarks. (1) Theorem 1, its corollary and Theorem 3 were known essentially for the classical groups (see $[6 ; 8 ; 9]$ ). Theorem 3 for algebraic tori is proved in [5]. As is known, it is easy to derive from them similar results on groups of matrices with coefficients in the ring of integers of a number field $K$, which belong to an algebraic group defined over $K$.

(2) Theorem 3 and known properties of semi-simple Lie algebras imply easily that a connected semi-simple Lie group $G$ always has discrete subgroups $H$ such that $G / H$ is compact.

${ }^{1}$ We understand that another proof of Theorem 3 has since been given by G. D. Mostow and T. Tamagawa. 


\section{BIBLIOGRAPHY}

1. A. Borel, Groupes linéaires algébriques, Ann. of Math. vol. 64(1956) pp. 20-80.

2. C. Hermite, Oeuvres complètes, Vol. 1, Paris, Gauthier-Villars, 1905.

3. Y. Matsushima, Espaces homogènes de Stein des groupes de Lie complexes, Nagoya Math. J. vol. 16 (1960) pp. 205-218.

4. G. D. Mostow, Self-adjoint group, Ann. of Math. vol. 62 (1955), pp. 44-55.

5. T. Ono, Sur une propriêté arithmétique des groupes algébriques commutatifs, Bull. Soc. Math. France vol. 85 (1957) pp. 307-323.

6. K. G. Ramanathan, Unit of fixed points in involutorial algebras, Proceedings of the International Symposium on Algebraic Number Theory, Tokyo, 1955.

7. Séminaire S. Lie, Théorie des algèbres de Lie, Topologie des groupes de Lie, Paris, 1954-1955.

8. C. L. Siegel, Einheiten quadratischer Formen, Abh. Math. Sem. Univ. Hamburg vol. 13 (1939) pp. 209-239.

9. A. Weil, Discontinuous subgroups of classical groups, Notes, University of Chicago, 1958.

The Institute for Advanced Study AND

Columbia University

\section{SOME PROPERTIES OF ADELE GROUPS ATTACHED TO ALGEBRAIC GROUPS}

BY ARMAND BOREL

Communicated by Deane Montgomery, July 22, 1961

This note is a sequel to the previous one [1], and is devoted to some applications of the results of the latter to adele groups. The results are valid for linear algebraic groups defined over number fields, but this case is easily reduced to that of groups defined over $\mathbf{Q}[\mathbf{3}$, Chapter I], to which we shall limit ourselves for simplicity.

The notation of [1] is freely used. For the unexplained notions concerning adeles, see $[2 ; 3]$.

1. Adeles. Let $G$ be a connected algebraic linear group defined over Q. The adele group attached to $G$ is denoted by $G_{A}$. The group $G_{\mathcal{B}}$ is identified with the subgroup of principal adeles of $G_{A}$; it is discrete. We put

$$
G_{A}^{0}=G_{\mathrm{R}} \times \prod_{p \text { prime }} G_{Z_{\mathrm{p}}} \quad\left(\boldsymbol{Z}_{p} \text { : ring of } p \text {-adic integers }\right) .
$$

By definition, $G_{A}^{0}$, endowed with the product topology, is an open subgroup of $G_{A}$. The group $G$ is said to be of type $(F)$ if $G_{A}$ is the union of a finite number of double cosets $G_{A}^{0} \cdot x \cdot G_{Q}\left(x \in G_{A}\right)$ [2]. 